\title{
Multi-relational Social Networks in a Large-scale MMORPG
}

\author{
Seokshin Son \\ Seoul National University \\ ssson@mmlab.snu.ac.kr \\ Ted "Taekyoung" Kwon \\ Seoul National University \\ tkkwon@snu.ac.kr
}

\author{
Ah Reum Kang \\ Korea University \\ armk@korea.ac.kr \\ Juyong Park \\ Kyunghee University \\ perturbation@gmail.com
}

\author{
Hyun-chul Kim \\ Seoul National University \\ hyunchulk@gmail.com \\ Huy Kang Kim \\ Korea University \\ cenda@korea.ac.kr
}

\begin{abstract}
We analyze multi-relational social interaction networks in a large-scale commercial Massively Multiplayer Online RolePlaying Game (MMORPG). Our work is based on data from AION, currently the world's second most-played MMORPG with 3.4 million subscribers as of mid 2010, created and serviced by NCSoft, Inc. We construct and characterize six distinct interactivity networks (Friend, Private Messaging, Party invitation, Trade, Mail, and Shop), each representing diverse player interaction types.
\end{abstract}

\section{Categories and Subject Descriptors}

J.4 [Computer Applications]: Social and Behavioral Sciences

\section{General Terms}

Human Factors, Measurement

\section{Keywords}

Social network analysis, Quantitative social science, Massively multiplayer online game

\section{INTRODUCTION}

It has been reported that more than 15 million people worldwide enjoy MMORPGs [1] that feature online social arenas in fantastical settings where gamers can engage in individual or group battles, exchange or trade items, or simply socialize with one another via in-game communication channels. Given that the complexity and variety of in-game interactions may now rival those of real-world interactions, the digital archive of online activities opens up an attractive avenue to researchers for a deep understanding of human social behaviors. Yet, such research is often hindered by the lack of access to the data. In this work we investigate multirelational social and collective aspects of MMORPG players' interactions based on data collected from $\mathrm{AION}^{1}$, the world's second largest MMORPG from NCSoft, Inc. Specifically, we construct and charactersize six distinct interactivity networks, each representing diverse player interaction types. To the best of our knowledge, this is the first work that analyzes

\footnotetext{
${ }^{1}$ AION Online, http://www.aiononline.com
}

Copyright is held by the author/owner(s). multi-relational social networks in a large-scale commercial MMORPG

Our study is based on the anonymized database dumps from one AION server/world out of 41 identically designed but independently running parallel game worlds/servers. The dataset includes all in-game interactions between player characters for 87 days, in mid 2010. Each interaction record contains anonymized IDs of the sender and receiver, and the type of the interaction record, most of which fall into one of the following six categories: (i) Friend F: A directed edge is created from user A to B when A adds B to A's buddy list. (ii) Whisper $\mathbf{W}$ : A directed edge is created from user A to B when A sends a private message to B. (iii) Party Invitation $\mathbf{P}$ : A directed edge is created from user A to user B when A invites B to join his party. A party is a group of up to six players that can carry out missions or engage in battles as a team. (iv) Trade $\mathbf{T}$ : A directed edge is formed from $\mathrm{A}$ to $\mathrm{B}$ when $\mathrm{A}$ requests a trade of items to $\mathrm{B}$, then $\mathrm{B}$ accepts it. (v) Mail $\mathbf{M}$ : An edge is formed from user A to B when A sends a mail to B. (vi) Shop S: users can enter a private market mode, becoming a merchant (and unable to do any other actions while in the mode) who can announce publicly the list of items for sale. Other users can then buy items from the merchant using in-game currency, in which case a directed edge is drawn from the buyer to the merchant.

\section{RESULTS}

Table $1^{2}$ shows how many gamers were engaged in each interaction type, and the number of interactions (for simplicity, we have not considered weights on the edges, which is the frequency of the interactions between user pairs). We see that the Party invitation, a truly social activity, is the most prevalent interaction while Shop is the least so. Note that the Giant Connected Components (GCCs) [2] take up approximately $50 \%$ of the nodes and the edges across the networks, while the second largest connected components (not shown) are very similar in size. While this phenomenon would be extremely unusual, it is expected in AION, since it features a Realm-vs-Realm design where a player belongs to either of two major tribes (Heavenly and Diabolical) that cannot communicate with each other. The clustering coefficients [2] in the networks are shown in Fig. 1(a), where Trade, Party, Whisper, and Friend networks exhibit clustering coefficients $C>0.03$, higher than others. In Fig. 1(b), we show by how many factors they are larger than expected

${ }^{2}$ Due to space limitations, we refer readers to [2] for the definitions of all the graph measures used. 
Table 1: Summary of the basic network characteristics.

\begin{tabular}{|l|c|c|c|c|c|c|}
\hline & Party(P) & Friend(F) & Whisper(W) & Trade(T) & Mail(M) & Shop(S) \\
\hline \# of Nodes & 45,590 & 29,995 & 20,107 & 45,567 & 56,040 & 9,423 \\
\hline \# of Edges & 910,171 & 103,437 & 176,245 & 179,277 & 170,774 & 18,882 \\
\hline \% of Nodes in GCC [2] & $47.44 \%$ & $46.83 \%$ & $50.24 \%$ & $47.06 \%$ & $49.34 \%$ & $51.17 \%$ \\
\hline \% of Edges in GCC [2] & $49.32 \%$ & $49.47 \%$ & $47.80 \%$ & $49.12 \%$ & $51.24 \%$ & $58.38 \%$ \\
\hline Network Diameter [2] & 15 & 15 & 11 & 27 & 13 & 13 \\
\hline Avg. Path Length [2] & 3.90 & 4.80 & 3.81 & 5.70 & 7.63 & 6.85 \\
\hline Avg. Degree [2] & 43.8 & 13.5 & 26.2 & 9.6 & 6.8 & 4.0 \\
\hline
\end{tabular}

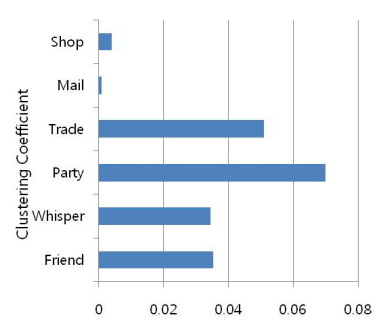

(a)

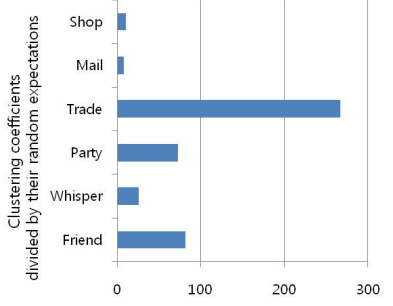

(b)
Figure 1: (a) The clustering coefficients $C$. (b) Clustering coefficients divided by their expected values in randomized networks of identical sizes.

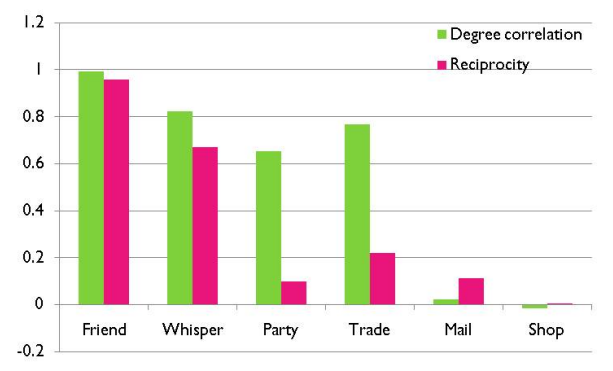

Figure 2: The Pearson correlation coefficients between the in- and the out-degrees of nodes, and the reciprocity of the interactions.

in randomized networks with the same number of nodes and edges. Again, we see that the four social-type networks (F, $\mathrm{W}, \mathrm{P}, \mathrm{T})$ show factors of 20 or larger indicating the strong clustering tendency.

The Pearson correlation coefficients [3] between the inand the out-degree of each node, and the reciprocity [2] of interactions are shown in Fig. 2. First, we see that in the four social-type networks $(\mathrm{F}, \mathrm{W}, \mathrm{P}, \mathrm{T})$ the in- and the outdegrees of nodes are high correlated (green, 0.6 or higher); if a user has a long buddy list, then they are likely to be in many buddy lists themselves, and people engaging in Party or Trade initiation are also likely to be actively invited to Parties and Trade. Again, Shop and Mail show very small in- and out-degree correlation. The case of Shop is due to the nature of the interaction and the preferred role that users take: Since a high in-degree represents the role of a merchant, the low correlation means that users who prefer to be one is seldom a buyer from a merchant, and vice versa. In Mail, on the other hand, we found that spammers who send many mails indiscriminately and receive few have a large effect on the statistics.
When we inspect the reciprocity, however, only two of the social-type networks ( $\mathrm{F}$ and $\mathrm{W}$ ) are highly reciprocated, while the other two ( $\mathrm{P}$ and $\mathrm{T}$ ) are generally not. This presents an interesting observation of the two interactions: when one invites others to a Party, becoming a "Party Leader", it is not necessarily the case that the invitee will at some point invite the original initiator to join their Party. Also, one who initiates a Trade will not necessarily be invited to a Trade by the same person. It indicates that a preferred partner of Party and Trade activities in a gamer's mind tends not to reciprocate the feeling; perhaps the level of strategic thinking necessary for starting a Party or Trade for items is higher - it is conceivable that one may be more interested in joining stronger players when one goes out on a mission - than for Friend or Whisper activities that do not require as much strategizing.

Our current on-going work includes further in-depth investigation of degree distributions [2], triangular motif statistics [5], and the network link and node overlap [4], etc. We believe they will shed light on the correlations and overlap between different types of networks, which in turn would help us understand the organization principles of the social system. In thie paper we reported on the very first results from the analysis of massive interaction data from AION. We believe that there are many promising avenues for more detailed research, for understanding not only human social behavior themselves in a virtual world, but also offline behavior otherwise difficult or impossible to study.

\section{ACKNOWLEDGEMENT}

This work was funded by KHU-20110088 from Kyung Hee University, KRF-20110005499 from the Korean Research Foundation, the Korea Broadcasting Service, the ITRC support program NIPA-2011-C1090-1001-0004 of MKE/NIPA, and the NAP of Korean Research Council of Fundamental Science and Technology.

\section{REFERENCES}

[1] S. Hill. MMO Subscriber Populations. http:// www.brighthub.com/video-games/mmo/articles/ 35992.aspx, March 2011.

[2] M. Newman. The Structure and Function of Complex Networks. SIAM Rev., 45(2), 2003.

[3] J. Rodgers and W. Nicewander. Thirteen Ways to Look at the Correlation Coefficient. The American Statistician, 42(1), 1988.

[4] J. Onnela, J. Samamaki, J. Hyvonen, G. Szabo, M. Menezes, K. Kaski, A. Barabasi, and J. Kertesz. Analysis of a Large-scale Weighted Network of One-to-one Human Communication. New Journal of Physics, 9(6), 2007.

[5] R. Milo, S. Shen-Orr, S. Itzkovitz, N. Kashtan, D. Chklovskii, and U. Alon. Network Motifs: Simple Building Blocks of Complex Network. Science, 298(5594), 2002. 